\title{
Physiologic Assessment of Coronary Artery Disease by Cardiac Computed Tomography
}

\author{
Minisha Kochar, MD'1 and James K. Min, MD²,3 \\ 'Division of Cardiology, Kaiser Permanente, Panorama City, CA, \\ ${ }^{2}$ Cedars-Sinai Heart Institute, Cedars-Sinai Medical Center, Los Angeles, CA, \\ ${ }^{3}$ Department of Medicine, David Geffen School of Medicine, University of California, Los Angeles, CA, USA
}

Coronary artery disease (CAD) remains the leading cause of death and morbidity worldwide. To date, diagnostic evaluation of patients with suspected CAD has relied upon the use of physiologic non-invasive testing by stress electrocardiography, echocardiography, myocardial perfusion imaging (MPI) and magnetic resonance imaging. Indeed, the importance of physiologic evaluation of CAD has been highlighted by large-scale randomized trials that demonstrate the propitious benefit of an integrated anatomic-physiologic evaluation method by performing lesion-specific ischemia assessment by fractional flow reserve (FFR)-widely considered the "gold" standard for ischemia assessment-at the time of invasive angiography. Coronary CT angiography (CCTA) has emerged as an attractive non-invasive test for anatomic illustration of the coronary arteries and atherosclerotic plaque. In a series of prospective multicenter trials, CCTA has been proven as having high diagnostic performance for stenosis detection as compared to invasive angiography. Nevertheless, CCTA evaluation of obstructive stenoses is prone to overestimation of severity and further, detection of stenoses by CCTA does not reliably determine the hemodynamic significance of the visualized lesions. Recently, a series of technological innovations have advanced the possibility of CCTA to enable physiologic evaluation of $C A D$, thereby creating the potential of this test to provide an integrated anatomic-physiologic assessment of CAD. These advances include rest-stress MPI by CCTA as well as the use of computational fluid dynamics to non-invasively calculate FFR from a typically acquired CCTA. The purpose of this review is to summarize the most recent data addressing these 2 physiologic methods of CAD evaluation by CCTA. (Korean Circ J 2013;43:435-442)

KEY WORDS: Prognosis; Coronary artery disease; Multiditector computed tomography.

\section{Introduction}

Coronary artery disease (CAD) is the leading cause of death in this world. In the United States alone, 785000 people have a new coronary event each year with an estimated direct and indirect cost of $\$ 110$ billion. ${ }^{122)}$ Regrettably, the initial diagnosis of CAD often occurs only after an event yields significant disability or worse, sudden car-

Correspondence: James K. Min, MD, Department of Medicine, Imaging, and Biomedical Sciences, Cedars-Sinai Heart Institute, Cedars-Sinai Medical Center, 8700 Beverly Boulevard, S. Mark Taper Building, Room 1253, Los Angeles, California 90048, USA

Tel: 1-310-423-2707, Fax: 1-310-423-0811

E-mail: james.min@cshs.org

- The authors have no financial conflicts of interest.

This is an Open Access article distributed under the terms of the Creative Commons Attribution Non-Commercial License (http://creativecommons. org/licenses/by-nc/3.0) which permits unrestricted non-commercial use, distribution, and reproduction in any medium, provided the original work is properly cited. diac death. Thus, early detection of CAD is imperative to determine optimal patient-specific therapies to reduce morbidity and mortality and thereby contain the ever-increasing medical and economic costs associated with this disease.

To date, the gold standard for the diagnosis of anatomic CAD has been invasive coronary angiography (ICA), which utilizes X-ray visualization of a radiopaque dye to detect the percentage of occlusion of a coronary vessel. ${ }^{3 / 4)}$ This method, while the standard diagnostic tool, is costly and subjects patients to non-negligible procedural risks. Indeed, a recent study from the American College of Cardiology National Cardiovascular Data Registry (NCDR) demonstrated that up to two-thirds of patients undergoing invasive testing by angiography are found not to have anatomically obstructive CAD. ${ }^{5)}$ This study, comprising 398978 patients at 663 hospitals, also determined that almost $40 \%$ of individuals referred for invasive angiography were found to have normal coronary arteries, despite non-invasive testing being performed in more than 4 of 5 individuals prior to ICA. These findings have evoked concerns that current non-inva- 
sive strategies are inadequate to properly identify individuals who are suitable for further invasive angiographic assessment.

\section{Coronary CT Angiography}

Coronary CT angiography (CCTA) has emerged as a novel non-invasive method for angiographic assessment of $C A D$ and proponents of CCTA have advocated for its use as an effective 'gatekeeper' to ICA procedures. The emergence of clinical application of CCTA coincided with the introduction of 64-detector row CCTA scanners in 2005. ${ }^{6}$ These CT scanners represented a transformational change in CT imaging-with generally high spatial and temporal resolution, and sufficient volume coverage to allow for whole heart image acquisition within a single breathhold. Early in the evolution of data development for CCTA, most studies focused primarily on the diagnostic accuracy of CCTA compared to ICA for the detection and exclusion of anatomically obstructive CAD, defined at the $50 \%$ or $70 \%$ threshold. While these initial studies were generally limited to single centers, small study samples and referral/selection biases, they nevertheless provided a "proof of principle" that CCTA detection of CAD demonstrated high diagnostic performance when compared to ICA. ${ }^{7-12)} A$ recent meta-analysis of these studies reported a sensitivity, specificity, positive predictive value (PPV), and negative predictive value (NPV) of 97\%, 90\%, 93\%, and 96\%, respectively for CCTA in quantifying CAD using ICA as the reference standard. ${ }^{13)}$

To address the biases of earlier studies, a series of prospective multicenter studies were performed to more objectively evaluate CCTA diagnostic performance. The first of these studies was performed in an intermediate pre-test likelihood patient population of stable symptomatic individuals without known CAD being referred to ICA. The ACCURACY study, or Assessment by Coronary Computed Tomographic Angiography for Individuals UndeRgoing InvAsive Coronary AngiographY, comprised 230 patients from 16 centers who underwent CCTA prior to ICA. Importantly, CCTAs were performed as part of the investigation rather than clinical care, thereby disencumbering the study of referral and/or selection bias. ${ }^{14)}$ At an intermediate prevalence of CAD that paralleled those observed by the NCDR registry, the sensitivity and NPV of CCTA approached 100\%, indicating a very low rate of false negative studies. In this regard, proponents of CCTA advocated for its use as an exclusionary non-invasive tool for identifying individuals who do not need to proceed to ICA. Yet, the ACCURACY study revealed a specificity of CCTA of 83\%, suggesting that there remains a non-negligible proportion of falsepositive studies which nevertheless continue to drive unnecessary ICA, albeit at a significantly reduced rate than has been reported before. $^{9)}$

Subsequent to the ACCURACY study, there have been at least $2 \mathrm{ad}-$ ditional prospective multicenter trials that have evaluated CCTA compared to ICA, and have uniformly demonstrated that, in patients without known CAD, CCTA excels for exclusion of obstructive CAD compared to other tests and correctly identifies obstructive $C A D$ in a manner similar to conventional stress testing modalities. ${ }^{1015)}$

\section{Limitations of Current Generation Coronary CT Angiography}

One consistently observed limitation of CCTA lies in overestimation of the presence and extent of $C A D$, which may significantly increase the rates of false-positive studies. ${ }^{6)}$ This occurs for a variety of reasons but is often due to acquisition artifact or blooming artifact from calcium deposition in a portion of the vessel. ${ }^{16-18)}$ At worst, this can precipitate unnecessary ICA and coronary revascularization in patients who do not have high-grade obstructive coronary stenoses or ischemia. ${ }^{5 / 6)}$ The ACCURACY trial examined the diagnostic performance of CCTA in patients with varying amounts of calcium, stratified into two groups by $<400$ and $>400$ Agatston units by coronary artery calcium (CAC) scoring. A higher CAC resulted in no changes to diagnostic sensitivity. However, a significant reduction in specificity for the diagnosis of obstructive CAD was found in the presence of higher CAC. The patient-based sensitivity for the presence of $>50 \%$ stenosis for patients with calcium scores $<400$ vs. $>400$ was $95.8 \%$ vs. 93.6\%, respectively; while the specificity of CCTA-diagnosed obstructive disease at the 50\% stenosis threshold was reduced in patients with CAC scores $>400$ vs. $<400$, at $52.6 \%$ vs. $86.3 \%$, respectively.9) The potential overestimation of CAD was most pronounced in lesions with $>50 \%$ stenosis--arguably the most clinically relevant lesions--with a 49\% accuracy rate. This reduced diagnostic accuracy may precipitate clinical uncertainty, and may lead to challenging management decisions where further tests such as myocardial perfusion testing or ICA may be necessary to better quantify the coronary disease as well as the functional significance of individual plaques. ${ }^{19)}$

When determining the anatomic estimation of individual coronary lesions, ICA is less affected by the artifacts of image acquisition or calcium blooming as found in CCTA but, nevertheless, has its own limitations. The angiogram is a visual estimate of the degree of stenosis of individual plaques utilizing healthy portions of the same coronary vessel to serve as the reference size. ${ }^{3 / 4)}$ This visual quantification can, at times, be difficult to determine when large portions of the vessel are diseased or when the vessel has remodeled over time as described by Glagov et al. ${ }^{20211}$ It is in these cases that a functional test may have significant utility to guide further management. One of these tests used during the time of ICA is fractional flow reserve (FFR) which has the ability to assess the functional significance 
of individual lesions. ${ }^{22}$

Fractional flow reserve is widely considered the gold standard physiologic test for assessment of lesion-specific ischemia. ${ }^{1823)} \mathrm{In}$ comparison to stress testing methods that identify either global myocardial ischemia or territory specific ischemia, FFR provides an added advantage of assessing ischemia at the lesion level, thereby providing the clinician with important information regarding the hemodynamic significance of specific stenoses. Indeed, when compared to the FFR reference standard, stress testing methods such as myocardial perfusion imaging (MPI) perform generally poorly. While these tests may be adequate for ischemia detection on a per-patient basis, they demonstrate poor discrimination of specific vessels that accommodate coronary lesions that cause ischemia. When examined against an anatomic standard of ICA-confirmed stenosis for per-vessel diagnostic performance, the sensitivity and specificity of MPI for the left anterior descending, left circumflex and right coronary arteries are only $80 \%$ and $70 \%, 70 \%$ and $76 \%$, and $63 \%$ and $73 \%$, respectively. ${ }^{24)}$ Further, when employing an FFR standard for vessel-specific ischemia among patients with multivessel CAD, MPI identifies ischemic territories correctly less than 50\% of the time, with both under- and overestimation of vessel-specific ischemia in $36 \%$ and $22 \%$ of cases, respectively. ${ }^{25}$

The FFR technique uses a pressure sensitive catheter to assess the rate of maximal myocardial blood flow through a diseased portion of an artery relative to the flow through a normal portion of the aorta in a hyperemic state. ${ }^{1826-28)}$ The FFR is considered diagnostic of ischemia at values $<0.80$, a threshold that can guide judicious use of coronary revascularization procedures. ${ }^{18)}$ The FAME trial showed that FFR-guided revascularization in patients with $>50 \%$ stenosis of lesions resulted in lower rates of stent placement, death, myocardial infarction and repeat revascularization in 1 year relative to visual estimation from ICA alone. In addition, FFR-guided revascularization resulted in decreased contrast use and resulted in a similar, if not improved, functional status with no decrease in health-related quality of life. Also of significance was the decreased procedure-related cost in FFR-guided revascularization. ${ }^{18)}$ The recently published FAME2 trial corroborated the results of the initial FAME study, demonstrating that in patients with stable angina and functionally significant lesions by FFR, percutaneous coronary intervention with optimal medical therapy is superior to optimal medical therapy alone. There was an increase by a factor of 8-fold in the need for urgent revascularization in the medical therapy alone group. This further stresses the importance of accurately quantifying the percentage of stenoses and their hemodynamic significance. ${ }^{29)}$

The demand to assess the functional significance of individual lesions and thereby the perfusion of the myocardium non-invasively exists and have led to advances within the field of CCTA. Two meth- ods used to evaluate the significance of CAD include the evaluation of FFR from static CT images as well as perfusion of the myocardium utilizing myocardial computed tomography perfusion (CTP) technology. Both of these techniques can be combined with the anatomic visualization of the coronary vessels from the CCTA to provide a more comprehensive picture of a patient's CAD.

\section{Non-Invasive Fractional Flow Reserve Derived from CT}

Recently, investigators have demonstrated the feasibility of computing FFR from typically-acquired static CT images (FFR CT $_{\text {) }}$ (Fig. 1). This technique employs allometric scaling laws to relate "form to function"; that is, to utilize the anatomic data from CT to determine the physiologic milieu that is present around the anatomy through computational fluid dynamic modeling. ${ }^{30)}$ This is a novel use of an existing technology which has previously been used in automobile and aeronautical engineering and enables prediction of the hemodynamic significance of a coronary artery lesion by generating pressure fields utilizing the concentration of contrast proximal and distal to a lesion visualized on $\mathrm{CT}^{31-33)}$ Briefly, blood is modeled as a Newtonian fluid with incompressible Navier-Stokes equations and solved subject to appropriate initial and boundary conditions with a finite element method on a parallel supercomputer. ${ }^{33 / 34)}$ The information obtained allows for computation of blood flow both at rest and in the hyperemic state in a manner similar to invasive FFR, but can be done in a non-invasive manner without additional medication administration or the associated procedural risks and additional radiation in a patient already undergoing cardiac CT.9) This "functional data" can be used in conjunction with the anatomic evaluation of vessels of standard CT images to help guide clinical decision-making, with hopes of improving event-free survival and reducing unnecessary revascularization. If proven, these methods may be effective in reducing health-care costs and obviating the need for further testing with associated procedural and radiation risk. $^{35136)}$

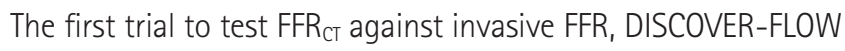
(Diagnosis of Ischemia-Causing Coronary Stenoses by Noninvasive FFR Computed from Coronary Computed Tomographic Angiograms), was a prospective multicenter evaluation of 103 stable patients from 4 centers with known or suspected CAD who underwent CT, ICA and FFR. Obstructive CAD by CCTA was defined as $>50 \%$ coronary artery luminal diameter stenosis and lesion specific ischemia was defined as an FFR or FFR $R_{C T}<0.80$. Compared to CCTA obstructive

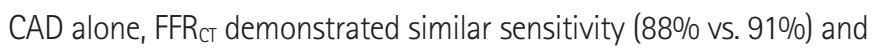
NPV (92\% vs. 89\%), but there was significantly higher specificity (82\% vs. 40\%) and PPV (74\% vs. 47\%) versus CT alone. This resulted 


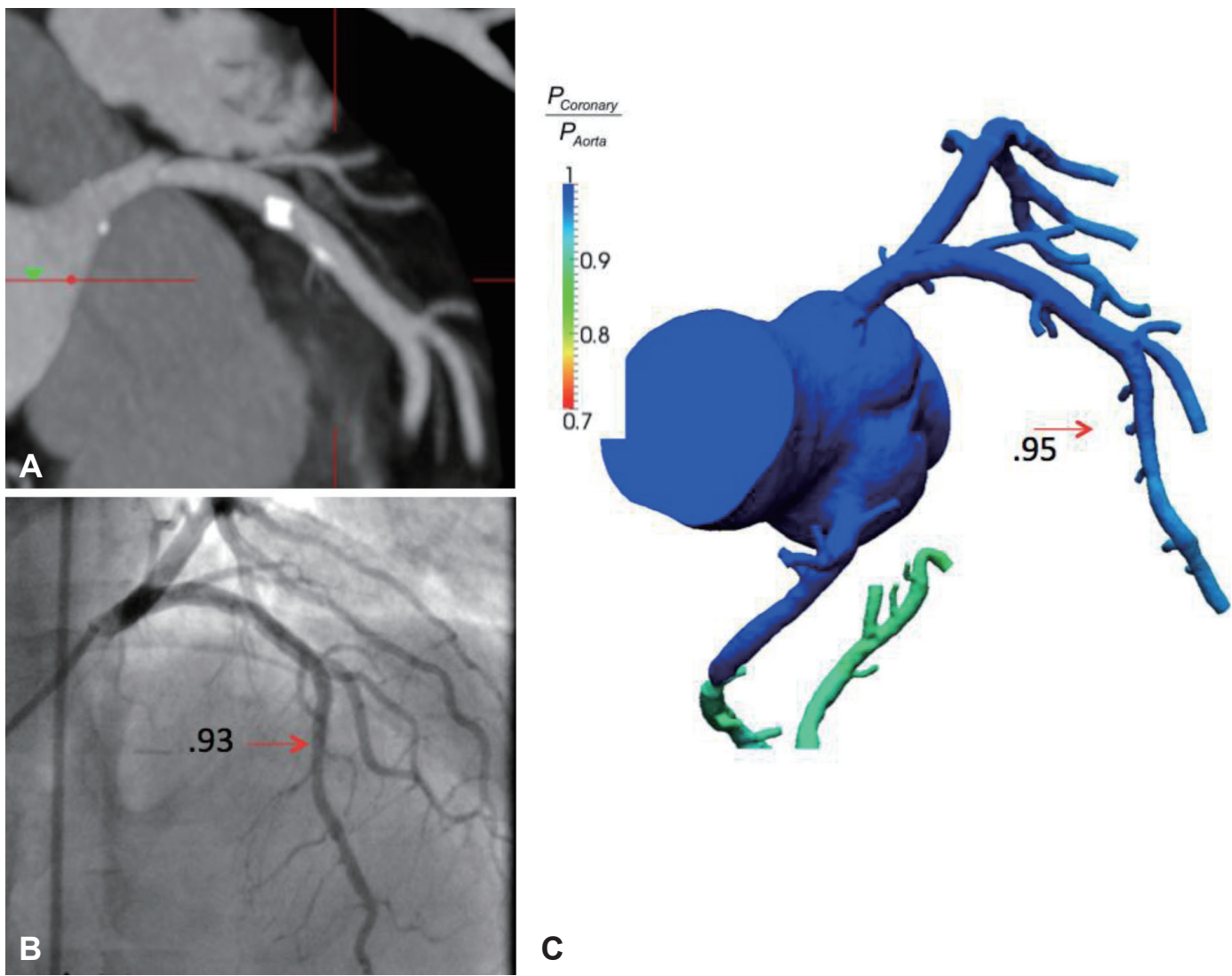

Fig. 1. Coronary CT angiogram, invasive coronary angiogram and FFR proximal LAD by CT. B: no significant stenosis or ischemia (FFR value 0.93) of the LAD by invasive angiography. C: FFR ischemia in the vessel (FFR ct value 0.95). FFR: fractional flow reserve.

in a 25\% overall improvement in overall diagnostic accuracy of FFR over CT alone (84\% vs. 59\%). Further, this study demonstrated that $\mathrm{FFR}_{\text {CT }}$ was superior and additive to $\mathrm{CT}$ for detecting stenoses for ischemia-causing lesions, largely by reducing the rates of false positive lesions as characterized by visual estimation on CT alone. ${ }^{22)}$

The subsequently published DeFACTO trial sought to assess the diagnostic performance of FFR in addition to CT for the diagnosis of hemodynamically significant coronary stenoses. This multicenter trial evaluated 252 patients from 17 centers in 5 countries with known or suspected CAD who underwent CCTA, ICA, FFR and FFR with the primary outcome assessing the accuracy of $\mathrm{CT}$ and FFR $\mathrm{FT}_{\mathrm{CT}}$ compared with the invasive FFR standard. Among the 137 patients with an abnormal FFR $(<0.80)$, the diagnostic accuracy, sensitivity, specificity, PPV, and NPV of FFR $R_{\text {ct }}$ were $73 \%, 90 \%, 54 \%, 67 \%$ and $84 \%$, respectively. When compared with CT alone, FFR ct was associated with improved discrimination of hemodynamically significant lesions. The study also demonstrated that FFR $\mathrm{R}_{\mathrm{ct}}$ improved discrimination of ischemia-causing stenoses relative to $\mathrm{CT}$ alone. ${ }^{9)}$

One limitation of FFR $\mathrm{Cr}$ as with the anatomic interpretation of CAD on CTA, interpretation is limited by the quality of image acquisition. This however will improve with better acquisition techniques and ongoing clinical trials are continuing to validate the high diagnostic performance of this technology. At this time this technology has not replaced FFR during ICA however the information acquired by FFR $R_{\text {CT }}$ may be used in conjunction with the anatomic data from the CTA images to engage the lower and intermediate-risk patient in discourse regarding further therapy.

\section{CT Myocardial Perfusion}

A second method of non-invasively assessing the severity of CAD is to determine the endpoint of coronary artery blood flow that is myocardial perfusion. The idea of CTP for evaluating myocardial perfusion in a clinical setting began 20 years ago with early generation electron beam CT scanners. However, CTP has been limited in its performance due to an array of technological obstacles; most notably, including radiation exposure to patients and acquisition artifacts such as beam hardening. ${ }^{37)}$

Myocardial CTP utilizes contrast enhancement within the myocardium to determine perfusion of tissue (Fig. 2). There are two methods used to determine the degree and distribution of contrast enhancement using both a qualitative and quantitative approach. Qu- 


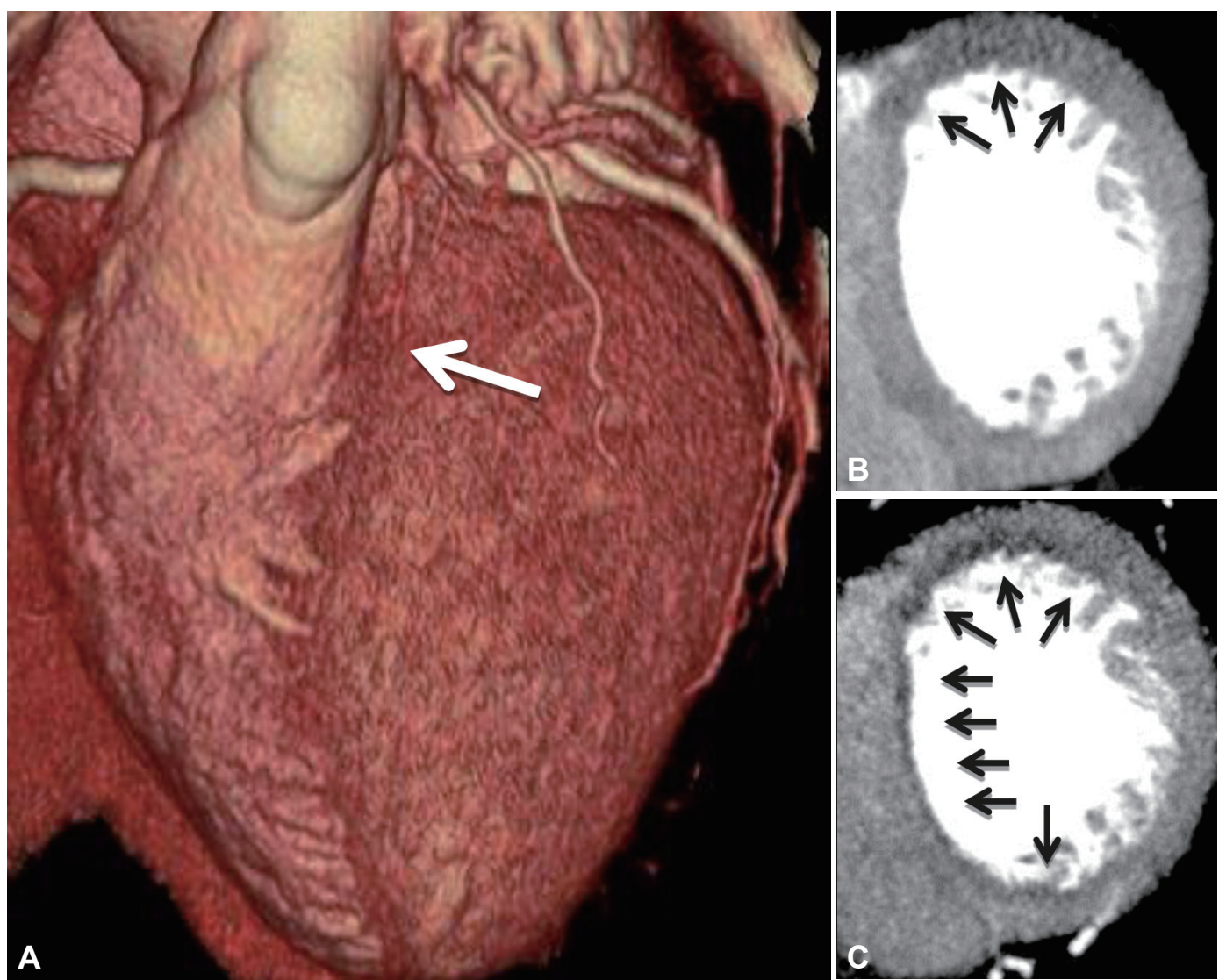

Fig. 2. Example of a CT myocardial perfusion study. A: volume rendered CT image demonstrating an atretic left anterior descending artery (white arrow). B: rest CT perfusion demonstrating hypoattenuation of the basal anterior wall (black arrows). C: stress CT perfusion after administration of regadenason demonstrating anterior and subendocardial anteroseptal inferoseptal and inferior ischemia (black arrows).

alitative myocardial CTP utilizes a visual perception of contrast density in different regions within the myocardium to distinguish normal from hypoperfused tissue. ${ }^{33388}$ Visual determination of contrast enhancement of the tissue can isolate regions of the myocardium with varying concentrations corresponding to perfusion from different coronary arteries. This method has been shown to reasonably distinguish infarction and exposes the patient to no additional radiation, however the ability to detect hypoperfusion is largely dependent on the timing of the $\mathrm{CT}$ acquisition. The acquisition of myocardial perfusion images must be done during peak myocardial contrast enhancement. This is, however, not always identical to the peak arterial contrast enhancement which is the typical protocol used in coronary CT. This time frame can also be delayed in ischemic or infarcted myocardium. ${ }^{39}$

An alternative quantitative CTP method provides a more accurate estimation of $C A D$, particularly in cases with left main or $3 \mathrm{~V}$ stenoses and acquires images throughout the arterial filling phase and the perfusion phase. Software has been developed to assess the distribution of contrast CT attenuation within the myocardium with a Gaussian distribution. Areas of hypodensity can be identified by statistical abnormalities in CT attenuation (Hounsfield units) from this model. ${ }^{40)}$

Nevertheless, beam hardening is a challenge in both quantitative and qualitative myocardial CTP. As the X-rays in the heart are attenuated to different extents based on the concentration of contrast in different chambers, $X$-ray photons with lower energies are preferentially removed. During the image reconstruction, projection measurements from all views are used to generate the entire image. These inconsistencies in X-ray images produce artifacts in the reconstructed images that typically appear as interspersed dark and light bands. With advances in CT technology using multiple X-ray sources and improvement in reconstruction algorithms, the beamhardening artifact may be minimized. ${ }^{32)}$ Recent investigations employing dual energy CT have demonstrated significant reductions in beam hardening artifact while allowing for absolute quantification of blood flow. ${ }^{411}$

Computed tomography perfusion studies in the setting of pharmacologic stress tests have been reported since 2009 with images being acquired during rest and then again after the administration of a pharmacologic stress agent, most commonly adenosine. While providing very useful information, the vasodilating agent adenosine is not identical to the effect of exercise on myocardial perfusion 
and therefore can not always mimic exercise-induced perfusion abnormalities. ${ }^{38) 42-44)}$ In addition, atherosclerosis limits the vasodilating effect of adenosine. In patients with severe stenosis and collateralization, adenosine may induce a coronary steal phenomenon thereby further decreasing contrast in the affected territory and giving the impression of less perfusion than is actually present. Critics of this method have thus contended that iodinated contrast in the myocardium may not actually represent the true myocardial blood flow. $^{45)}$

A recent study sought to evaluate the accuracy of resting CTP for the detection of perfusion defects compared against resting myocardial single photon emission computerized tomography (SPECT) images in 76 patients presenting to the early arterial phase defect with chest pain. The coronary CCTA anatomic information was also combined with the stress images obtained during the SPECT study and evaluated the accuracy for detecting $>50 \%$ stenoses on coronary CCTA. Of these cases, 13 were positive for resting perfusion defects by SPECT. Ten were due to ischemia and infarct, with the remainder due to chronic infarct. The diagnostic accuracy per patient was found to have 92\% sensitivity, 95\% specificity, 80\% PPV, and 98\% NPV. This showed that the addition of myocardial CTP to CCTA improved the accuracy of CCTA, primarily by reducing the rates of false-positive CCTA, thereby increasing the PPV. ${ }^{33)}$

The field of CTP is growing rapidly, with an array of studies evaluating its diagnostic performance, typically against a myocardial perfusion SPECT or cardiac magnetic resonance reference standard. Yet, similar to the early studies of CCTA, these studies have been limited to single centers with expert readers, small sample sizes and referral and/or selection biases. To date, no prospective multicenter study has yet been published to address these limitations. Recently, however, the CorE320 study, a prospective international investigation, was reported at the European Society of Cardiology 2012 Scientific Sessions. This study evaluated the accuracy of a combined metric of CCTA and CT perfusion against ICA with myocardial perfusion SPECT. When combining the CT angiographic findings with CTP for the detection of flow-limiting disease, the combined CTA/ CTP approach discriminated well with an area under the receiver operating characteristics curve of 0.87 on a per-patient basis. Another ongoing prospective multicenter study is underway to evaluate similar diagnostic endpoints (clinicaltrials.gov NCT01334918). While these early findings are exciting and suggest the potential of CT for stress perfusion assessment, they are nevertheless limited by the same confounders as those affecting standard stress imaging methods; namely, the relative nature of perfusion assessment, and the inability to precisely pinpoint the lesions that cause ischemia. Future studies examining the role of CTP to help guide decisions of revascularization are thus needed.

\section{Conclusions}

FFR $R_{C T}$ and myocardial CTP are novel methods recently developed for the physiologic assessment of CAD identified by CCTA. Data for $F_{\text {FF }}$ derived from prospective multicenter studies and data for CTP derived from single center cohorts have suggested their use as a complementary tool to CCTA to improve the specificity of coronary $\mathrm{CT}$ to identify hemodynamically significant CAD. These methods may encourage the use of CT as a "one stop shop" wherein anatomic stenosis severity and physiologic ischemia may be obtained in a single setting. Importantly, given the lack of need for additional testing, radiation or contrast, the use of FFR $\mathrm{CT}_{\text {T }}$ may be particularly useful for identifying the specific lesions that cause ischemia. These methods may play an essential role in discriminating individuals who may benefit versus not benefit from further testing by invasive angiography with intended coronary revascularization.

\section{References}

1. Roger VL, Go AS, Lloyd-Jones DM, et al. Heart disease and stroke statistics--2011 update: a report from the American Heart Association. Circulation 2011;123:e18-209.

2. Heidenreich PA, Trogdon JG, Khavjou OA, et al. Forecasting the future of cardiovascular disease in the United States: a policy statement from the American Heart Association. Circulation 2011;123:933-44.

3. Fischer JJ, Samady H, McPherson JA, et al. Comparison between visual assessment and quantitative angiography versus fractional flow reserve for native coronary narrowings of moderate severity. Am J Cardiol 2002;90:210-5.

4. Topol EJ, Nissen SE. Our preoccupation with coronary luminology. The dissociation between clinical and angiographic findings in ischemic heart disease. Circulation 1995;92:2333-42.

5. Patel MR, Peterson ED, Dai $D$, et al. Low diagnostic yield of elective coronary angiography. N Engl J Med 2010;362:886-95.

6. Min JK, Shaw $\sqcup$, Berman DS. The present state of coronary computed tomography angiography a process in evolution. J Am Coll Cardio/2010; 55:957-65.

7. Pugliese F, Mollet NR, Runza G, et al. Diagnostic accuracy of non-invasive 64-slice CT coronary angiography in patients with stable angina pectoris. Eur Radiol 2006;16:575-82.

8. Raff GL, Gallagher MJ, O'Neill WW, Goldstein JA. Diagnostic accuracy of noninvasive coronary angiography using 64-slice spiral computed tomography. J Am Coll Cardiol 2005;46:552-7.

9. Schuijf JD, Pundziute G, Jukema JW, et al. Diagnostic accuracy of 64-slice multislice computed tomography in the noninvasive evaluation of significant coronary artery disease. Am J Cardio/ 2006;98:145-8.

10. Ropers D, Rixe J, Anders K, et al. Usefulness of multidetector row spiral computed tomography with $64-\times 0.6-\mathrm{mm}$ collimation and 330-ms rotation for the noninvasive detection of significant coronary artery stenoses. Am J Cardio/ 2006;97:343-8.

11. Ehara M, Surmely JF, Kawai M, et al. Diagnostic accuracy of 64-slice 
computed tomography for detecting angiographically significant coronary artery stenosis in an unselected consecutive patient population: comparison with conventional invasive angiography. Circ J 2006;70: 564-71.

12. Nikolaou K, Knez A, Rist $C$, et al. Accuracy of 64-MDCT in the diagnosis of ischemic heart disease. AJR Am J Roentgenol 2006;187:111-7.

13. Hamon M, Biondi-Zoccai GG, Malagutti $P$, et al. Diagnostic performance of multislice spiral computed tomography of coronary arteries as compared with conventional invasive coronary angiography: a meta-analysis. J Am Coll Cardiol 2006;48:1896-910.

14. Min JK, Leipsic J, Pencina MJ, et al. Diagnostic accuracy of fractional flow reserve from anatomic CT angiography. JAMA 2012;308:1237-45.

15. Miller JM, Rochitte CE, Dewey M, et al. Diagnostic performance of coronary angiography by 64-row CT. N Eng/ J Med 2008;359:2324-36.

16. Meijboom WB, Meijs MF, Schuijf JD, et al. Diagnostic accuracy of 64-slice computed tomography coronary angiography: a prospective, multicenter, multivendor study. J Am Coll Cardio/ 2008;52:2135-44.

17. Budoff MJ, Dowe D, Jollis JG, et al. Diagnostic performance of 64-multidetector row coronary computed tomographic angiography for evaluation of coronary artery stenosis in individuals without known coronary artery disease: results from the prospective multicenter ACCURACY (Assessment by Coronary Computed Tomographic Angiography of Individuals Undergoing Invasive Coronary Angiography) trial. J Am Coll Cardiol 2008;52:1724-32.

18. Mowatt $G$, Cummins $E$, Waugh $N$, et al. Systematic review of the clinical effectiveness and cost-effectiveness of 64-slice or higher computed tomography angiography as an alternative to invasive coronary angiography in the investigation of coronary artery disease. Health Technol Assess 2008;12:iii-iv, ix-143.

19. Meijboom WB, Van Mieghem CA, van Pelt N, et al. Comprehensive assessment of coronary artery stenoses: computed tomography coronary angiography versus conventional coronary angiography and correlation with fractional flow reserve in patients with stable angina. $J$ Am Coll Cardiol 2008;52:636-43.

20. Glagov S, Bassiouny HS, Sakaguchi Y, Goudet CA, Vito RP. Mechanical determinants of plaque modeling, remodeling and disruption. Atherosclerosis 1997;131 Suppl:S13-4.

21. Glagov S, Weisenberg E, Zarins CK, Stankunavicius R, Kolettis GJ. Compensatory enlargement of human atherosclerotic coronary arteries. N Engl J Med 1987;316:1371-5.

22. Tonino PA, De Bruyne $B$, Pijls NH, et al. Fractional flow reserve versus angiography for guiding percutaneous coronary intervention. N Engl J Med 2009;360:213-24.

23. Pijls NH, De Bruyne B. Coronary pressure measurement and fractional flow reserve. Heart 1998;80:539-42.

24. Elhendy A, Schinkel AF, Bax JJ, et al. Accuracy of stress Tc-99m tetrofosmin myocardial perfusion tomography for the diagnosis and localization of coronary artery disease in women. J Nucl Cardiol 2006; 13:629-34.

25. Melikian N, De Bondt $P_{\text {, Tonino }}$, et al. Fractional flow reserve and myocardial perfusion imaging in patients with angiographic multivessel coronary artery disease. JACC Cardiovasc Interv 2010;3:307-14.

26. De Bruyne B, Baudhuin T, Melin JA, et al. Coronary flow reserve calcu- lated from pressure measurements in humans. Validation with positron emission tomography. Circulation 1994;89:1013-22.

27. Berger A, Botman KJ, MacCarthy PA, et al. Long-term clinical outcome after fractional flow reserve-guided percutaneous coronary intervention in patients with multivessel disease. J Am Coll Cardio/ 2005;46: 438-42.

28. Pijls NH, van Son JA, Kirkeeide RL, De Bruyne B, Gould KL. Experimental basis of determining maximum coronary, myocardial, and collateral blood flow by pressure measurements for assessing functional stenosis severity before and after percutaneous transluminal coronary angioplasty. Circulation 1993;87:1354-67.

29. De Bruyne B, Pijls NH, Kalesan B, et al. Fractional flow reserve-guided $\mathrm{PCl}$ versus medical therapy in stable coronary disease. N Engl J Med 2012;367:991-1001.

30. Koo BK, Erglis A, Doh JH, et al. Diagnosis of ischemia-causing coronary stenoses by noninvasive fractional flow reserve computed from coronary computed tomographic angiograms. Results from the prospective multicenter DISCOVER-FLOW (Diagnosis of Ischemia-Causing Stenoses Obtained Via Noninvasive Fractional Flow Reserve) study. J Am Coll Cardiol 2011;58:1989-97.

31. Kim HJ, Vignon-Clementel IE, Coogan JS, Figueroa $C A$, Jansen $K E_{1}$ Taylor CA. Patient-specific modeling of blood flow and pressure in human coronary arteries. Ann Biomed Eng 2010;38:3195-209.

32. Kim HJ, Jansen KE, Taylor CA. Incorporating autoregulatory mechanisms of the cardiovascular system in three-dimensional finite element models of arterial blood flow. Ann Biomed Eng 2010;38:2314-30.

33. Kim HJ, Vignon-Clementel IE, Figueroa $C A$, et al. On coupling a lumped parameter heart model and a three-dimensional finite element aorta model. Ann Biomed Eng 2009;37:2153-69.

34. Vignon-Clementel IE, Figueroa CA, Jansen KE, Taylor CA. Outflow boundary conditions for 3D simulations of non-periodic blood flow and pressure fields in deformable arteries. Comput Methods Biomech Biomed Engin 2010;13:625-40.

35. Fearon WF, Bornschein $B$, Tonino PA, et al. Economic evaluation of fractional flow reserve-guided percutaneous coronary intervention in patients with multivessel disease. Circulation 2010;122:2545-50.

36. Pijls NH, Fearon WF, Tonino PA, et al. Fractional flow reserve versus angiography for guiding percutaneous coronary intervention in patients with multivessel coronary artery disease: 2-year follow-up of the FAME (Fractional Flow Reserve Versus Angiography for Multivessel Evaluation) study. J Am Coll Cardiol 2010;56:177-84.

37. Wolfkiel CJ, Ferguson JL, Chomka EV, et al. Measurement of myocardial blood flow by ultrafast computed tomography. Circulation 1987; 76:1262-73.

38. George RT, Arbab-Zadeh A, Miller JM, et al. Adenosine stress 64- and 256-row detector computed tomography angiography and perfusion imaging: a pilot study evaluating the transmural extent of perfusion abnormalities to predict atherosclerosis causing myocardial ischemia. Circ Cardiovasc Imaging 2009;2:174-82.

39. So A, Lee TY. Quantitative myocardial CT perfusion: a pictorial review and the current state of technology development. J Cardiovasc Comput Tomogr 2011;5:467-81.

40. Feuchtner GM, Plank F, Pena C, et al. Evaluation of myocardial CT per- 
fusion in patients presenting with acute chest pain to the emergency department: comparison with SPECT-myocardial perfusion imaging. Heart 2012;98:1510-7.

41. So A, Hsieh J, Narayanan S, et al. Dual-energy CT and its potential use for quantitative myocardial CT perfusion. J Cardiovasc Comput Tomogr 2012;6:308-17.

42. Blankstein $R$, Shturman LD, Rogers IS, et al. Adenosine-induced stress myocardial perfusion imaging using dual-source cardiac computed tomography. J Am Coll Cardiol 2009;54:1072-84.
43. Feuchtner G, Goetti R, Plass A, et al. Adenosine stress high-pitch 128-slice dual-source myocardial computed tomography perfusion for imaging of reversible myocardial ischemia: comparison with magnetic resonance imaging. Circ Cardiovasc Imaging 2011;4:540-9.

44. Rocha-Filho JA, Blankstein $R$, Shturman $L D$, et al. Incremental value of adenosine-induced stress myocardial perfusion imaging with dualsource CT at cardiac CT angiography. Radiology 2010;254:410-9.

45. Williams MC, Newby DE. CT myocardial perfusion: a step towards quantification. Heart 2012;98:521-2. 